Obere Extremität 2018 $\cdot 13: 235-236$ https://doi.org/10.1007/s11678-018-0492-y Online publiziert: 13 . November 2018 (c) Springer Medizin Verlag GmbH, ein Teil von Springer Nature 2018

CrossMark

\section{Matthias A. Zumstein ${ }^{1}$. Bernhard Jost ${ }^{2}$}

' Universitätsklinik für Orthopädische Chirurgie und Traumatologie der Universität Bern, Inselspital Bern, Bern, Schweiz

${ }^{2}$ Klinik für Orthopädische Chirurgie und Traumatologie, Kantonsspital St. Gallen, St. Gallen, Schweiz

\title{
Irreparable posterosuperiore Massenrupturen der Rotatorenmanschette
}

Sehr geehrte Kolleginnen und Kollegen,

nebst dem Vorgehen der Diagnostik und der Therapie wird v.a. die Definition einer irreparablen posterosuperioren Massenrupturen der Rotatorenmanschette (IPSMR) kontrovers diskutiert.

Eine irreparable Ruptur bedeutet nicht eine Unmöglichkeit der chirurgischen Rekonstruktion, sondern vielmehr eine Unfähigkeit der Muskelsehneneinheit, ihre Aufgabe als „Motor“ zu bewerkstelligen. Als indirekte Messparameter der Irreparabilität bei chronischen Rupturen können hier

1. eine verminderte akromiohumerale Distanz von $<6 \mathrm{~mm}$ im Standard-

a.-p.-Röntgenbild in Neutralrotation,

2. eine Verfettung der posterosuperioren Rotatorenmanschette (RM) mit einem Stadium III/IV nach Goutallier,

3. eine Retraktion des Sehnenstumpfes bis respektive übers Glenoid und

4. eine Sehnenstumpflänge der rupturierten $\mathrm{RM}$ von $<15 \mathrm{~mm}$

genannt werden.

Obwohl verschiedene Autoren in der Definition der Massenruptur die Größe von $>5 \mathrm{~cm}$ als entscheidenden Faktor betrachten, halten wir uns aus Gründen der schwierigen Messgenauigkeit und -reproduzierbarkeit an die Definition eines kompletten Risses von mehr als 2 Sehnen von der Insertion am Humerus.

Eines der anspruchsvollsten Themen in der Schulterchirurgie stellt die Behandlung dieser symptomatischen IPSMR dar. Die Literatur beschreibt, dass nur unge- fähr $12 \%$ aller posterosuperioren RMRupturen irreparabel sind. Wenn diese symptomatisch werden, können sie mit verschiedenen konservativen und operativen Techniken behandelt werden. Oftmals fehlt jedoch die klare Evidenz hinter der möglicherweise sehr kostspieligen gewählten Therapie. Beispielweise werden komplette Rupturen des Supraund Infraspinatus, welche nur in einem niedrigen Prozentsatz richtig pseudoparetisch sind, je nach Einflussfaktoren wie Geographie, chirurgische Präferenz und Implantatkosten mit unterschiedlichen therapeutischen Verfahren wie Physiotherapie, subacromialem Débridement mit Bizepsbehandlung, partieller Rekonstruktion, Sehnentransfer des Latissimus dorsi (LDT), des tiefen Trapezius (tTRAP), einer superioren Kapselrekonstruktion (SCR), einem „subacromial spacer balloon" oder sogar einer inversen Schultertotalprothese behandelt. Oftmals werden diese unterschiedlichen Therapien, obwohl sie verschiedene Indikationen aufweisen, den gleichen Patienten mit der vermeintlich gleichen Diagnose nahezu willkürlich vorgeschlagen.

Deshalb ist es aus unserer Sicht von entscheidender Bedeutung und substanzieller Bestandteil dieser Ausgabe, diesem Aspekt Rechnung zu tragen. Nebst der klaren Definition einer IPSMR sollte das klinische Auftreten und Ausmaß einer allfälligen Pseudoparese in der entsprechenden Bewegungsamplitude Flexion, Außenrotation oder kombiniert analysiert und in den Entscheidungsalgorithmus einbezogen werden.
Es ist uns für diese Ausgabe gelungen, weltweit führende Referenten zu den entsprechenden Themen als Autoren für die $O b E x$ zu gewinnen, und wir denken ihnen mit den nachfolgenden Artikeln eine Übersicht der wichtigsten Aspekte der IPSMR geben zu können.

Dr. Plachel und Dr. Minkus aus der Arbeitsgruppe um Prof. Scheibel werden Ihnen ein Überblick über die aktuelle Datenlage, die Indikationsstellung und erste klinische und strukturellen Ergebnisse der SCR geben. Eine sehr innovative und interessante Technik des nahezu komplett arthroskopisch assistierten LDT wird ihnen durch die französische Gruppe von Dr. Kany, Dr. Anis und Dr. Werthel vorgestellt. Im Gegensatz zum klassischen LDT, welcher in einer partiell arthroskopischen Technik und mit Indikationsalgorithmus durch $D r$. Weihs vorgestellt wird, sind die tTRAP chirurgische Transfertechniken, welche insbesondere den Verlust der Außenrotation am Körper, der sog. Außenrotation 1, adressieren. Diese tTRAP stellen chirurgisch anspruchsvolle Techniken dar, welche mittels Autograftaugmentation mit Semitendinosus (im Artikel von $\mathrm{Dr}$. $\mathrm{Va}$ lenti) oder mittels Augmentation eines Allografts der Achillessehne (im Artikel von Dr. Aibinder und Prof. Elhassan) durchgeführt werden können. Die Studiengruppe aus der Mayo Klinik in Rochester, USA stellt ihre Therapieergebnisse nach den entsprechenden Eingriffen als Originalarbeit vor.

Zusammenfassend bleibt festzuhalten, dass verschiedene chirurgische Optionen in der Therapie der symptoma- 
tischen irreparablen posterosuperioren Massenrupturen der Rotatorenmanschette diskutiert werden. Es scheint kein Patentrezept in der Behandlung dieser Patienten zu geben, und es gilt in Betrachtung zu ziehen, dass im Falle einer palliativen oder nicht prothetischen Versorgung, auch mit aufwändigen materialintensiven Verfahren, wie beispielweise des SCR, mitunter hohe Versagerraten in bis $\mathrm{zu} 50 \%$ berichtet werden. Sowohl beim SCR als auch beim klassischen arthroskopisch assistierten LDT zeigt sowohl die Qualität und die Reparabilität der transversalen Rotatoren (Subskapularis und hypertropher Teres minor) einen positiven Effekt auf die klinischen und strukturellen Ergebnisse zu haben. Die gezeigten Artikel sowie auch verschiedene Übersichtsartikel in der Literatur rechtfertigen keine klaren Vorteile eines Verfahrens gegenüber einem anderen.

Wir wünschen ihnen viel Spaß bei der Lektüre dieses Themenheftes.

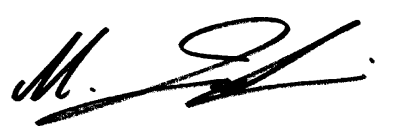

Prof. Dr. Matthias A. Zumstein

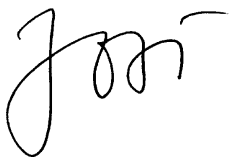

Prof. Dr. Bernhard Jost

\section{Korrespondenzadresse}

Prof. Dr.
Matthias A. Zumstein
Universitätsklinik für
Orthopädische Chirurgie und
Traumatologie der Universität
Bern, Inselspital Bern
3010 Bern, Schweiz
m.zumstein@insel.ch

Prof. Dr. Bernhard Jost
Klinik für Orthopädische
Chirurgie und Traumatologie,
Kantonsspital St. Gallen
9007 St. Gallen, Schweiz
Bernhard.Jost@kssg.ch

Interessenkonflikt. M.A. Zumstein und B. Jost sind Berater von Medacta International. 